\title{
A discrepancy between anticipated reward and obtained reward with no increase in resistance to extinction ${ }^{1}$
}

\author{
E. J. CAPALDI and ELIZABETH D. \\ CAPALDI, Purdue University, Lafayette, \\ Ind. 47907
}

One of two varied magnitude-of-reward groups, trained in a runway under conditions designed to preclude anticipation of the smaller reward magnitude, nevertheless failed to show increased resistance to extinction, a result consistent with the sequential hypothesis. Since this group must have experienced frustration or dissonance in acquisition, its failure to show increased resistance to extinction is inconsistent with those hypotheses. This result suggests either that frustration or dissonance is irrelevant in determining extinction performance or that both hypotheses have failed to specify and identify the conditions that are both necessary and sufficient for increasing resistance to extinction.

The purposes of this investigation were several, among them to determine if those hypotheses that place a critical emphasis on receiving a smaller reward magnitude than expected have identified the conditions that are both necessary and sufficient for increasing resistance to extinction ( $R$ to $E$ ). The means employed to test these views were complex but, it is believed, entirely adequate. While it is not widely recognized, the implications of sequence-of-reward data for "expectation-disconfirmation" viewpoints are, on the whole, unfavorable. Our specific starting point, however, is anticipated nonreward. By anticipated nonreward is meant nonrewarded trials on which $S$ once ran rapidly (presumably anticipating reward) but now runs slowly (presumably no longer anticipating reward). Examples exist in Capaldi (1958) and Mackinnon (1967).

Anticipated nonreward, it has been suggested (e.g., Lawrence \& Festinger, 1962), will not produce dissonance and thus will not increase $R$ to $E$. More specifically, this hypothesis has been employed to explain the results of sequence-of-reward investigations (e.g., Capaldi, 1958), which show that $R$ to $E$ will vary when number of nonrewards has been held constant. Such data are incompatible with Lawrence and Festinger's view that $R$ to $E$ is an increasing function of number of nonrewards. They begin by noting that in some sequence-of-reward investigations (e.g., Capaldi, 1958), certain groups began to anticipate nonreward by virtue of the regular reward schedules employed. In their view, these groups no longer experienced dissonance and, accordingly, $R$ to $E$ was independent of number of nonrewards. However, they suggest that, if reward were anticipated on nonrewarded trials, $R$ to $E$ would be independent of sequence of reward.

The anticipated-nonreward hypothesis was tested here by employing Leonard's (1969) varied magnitude-of-reward schedules. Leonard employed two trials a day, and small reward (S) either preceded or followed large reward (L), i.e., SL vs LS. In the present investigation, however, on some of the days, Group SL and Group LS were trained $\mathrm{LL}$. The purpose of the $\mathrm{LL}$ training was to forestall the anticipation of small reward on Trial 1 in Group SL and on Trial 2 in Group LS. A control group, trained LL on all days, was also included. There is no necessary suggestion here that in Leonard's investigation the reward schedules were anticipated. However, as indicated elsewhere (Capaldi, in press), the training procedures employed here allow one to have simultaneously a "regular" reward schedule within days, which satisfies some of the requirements of the sequential hypothesis, and an "irregular" schedule over days, which satisfies the requirements of theories of the dissonance and frustration types.

\section{METHOD}

The 36 male rats, about 90 days old on arrival at the laboratory, were purchased from the Holtzman Co., Madison, Wisconsin. The gray wood alley was $4 \mathrm{in}$. wide and enclosed by 9-in.-high sides covered with hinged $1 / 2$-in. hardware cloth. When a 10-in. start treadle was depressed by the rat, whose front paws were always placed on the treadle's extreme forward edge, the first clock $(.01 \mathrm{sec})$ started. Clock 1 was stopped (start time) and Clock 2 started when $S$ broke an infrared beam 4 in. beyond the treadle. Clock 2 was stopped (run time) and Clock 3 started when $S$ broke the second beam, located 52 in. from the first. The third infrared beam was $12 \mathrm{in}$. beyond the second beam (goal time) and 2 in. in front of a brass, $2 \times 4 \frac{1}{4} \times 1 \frac{112}{2}$ in. foodcup, covered by a sliding lid that opened automatically when the third beam was broken. When $S$ broke the third beam, a brass guillotine door,
12 in. from the alley's distal end was lowered inanually. The elapsed time on the three clocks was summed and is termed "total."

Experimental training followed 10 days of deprivation $(12 \mathrm{~g})$ and handling. Ss cxplored the apparatus for $5 \mathrm{~min}$ on Days 7 and 9 and received $10.045 \mathrm{~g}$ Noyes pellets in the home cage on Days 7-10. On each of the 15 acquisition days, there were two daily trials, reward being either large (L), 20.045 -g Noyes pellets, or small (S), 2 pellets. On Days 4, 5, 8, 12, and 13 of acquisition, all Ss received large reward on both acquisition trials (L,L). On the remaining 10 days of acquisition, Group SL received S on Trial 1 (T1) and $L$ on Trial 2 (T2), while Group $L S$ received $L$ on $T 1$ and $S$ on $T 2$. Group $L L$ received $L$ on $\mathrm{T} 1$ and $\mathrm{T} 2$ on all days. The Ss were run randomly within squads of three $S$, one $S$ from each group. For three of the squads, acquisition began on Day 11, for three more squads, on Day 12, etc. The in tertrial interval (ITI) in acquisition ranged from 2 to $7 \mathrm{~min}$, depending upon reward condition ( $L$ or $S$ ) and stage of training. On the day following acquisition training, there were 12 extinction trials, nonreinforced confinement duration being $30 \mathrm{sec}$. The ITI in extinction varied from 2 to $8 \mathrm{~min}$, depending upon stage of training. If a rat failed to leave an alley section within $60 \mathrm{sec}$, it was placed in the goalbox, and a time of $60 \mathrm{sec}$ was recorded for each untraversed section. Ss were fed the daily diet minus the amount received in the goalbox 10-15 min following the last trial of each day.

\section{RESULTS}

Acquisition

Differences between Groups SL, LS, and LL were not significant for total speed employing the last 3 days of acquisition $(\mathrm{F}<1)$. As a check on discrimination, an analysis was also performed comparing the $\mathrm{T} 1$ and $\mathrm{T} 2$ speeds of each group on the last 3 days of acquisition and on the last day of acquisition alone. None of these six differences approached significance, most $y$ ielding $\mathrm{F}<1$. Close inspection of individual $S$ data also provided no consistent basis for inferring that discrimination between $\mathrm{T} 1$ and $\mathrm{T} 2$ occurred in either Group SL or Group LS. For every SL or LS animal whose speeds differed either somewhat or considerably on $\mathrm{T} 1$ and $\mathrm{T} 2$, one could find an animal from Group LL that performed in a more or less similar fashion. As a final check on discrimination, the number of Ss whose speeds differed on $\mathrm{T} 1$ and $\mathrm{T} 2$, no matter how small the difference, in Groups SL and LS were compared. The conclusion from this comparison would be, if any thing, that Group SL discriminated better than did 


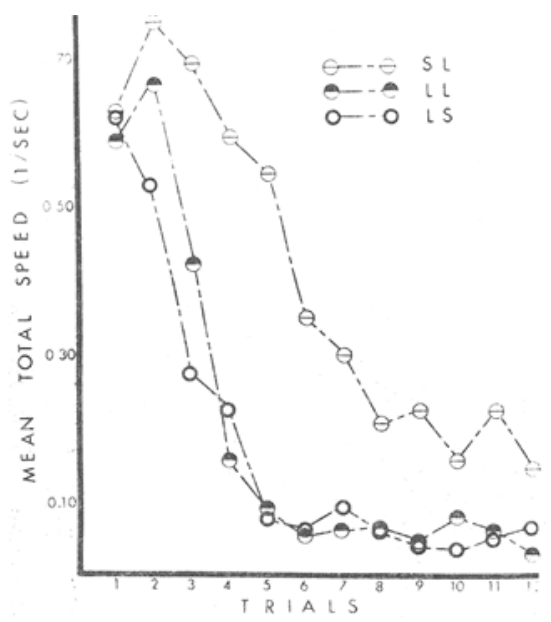

Fig. 1. Running speed of each of the three groups on each trial of extinction in total.

Group LS. On the last day of acquisition, 9 or $12 \mathrm{Ss}$ in Group SL were slower on T1 than on T2 (most of the differences, of course, being very small), while in Group LS, only 5 of 12 Ss were slower on $\mathrm{T} 2$ than on $\mathrm{T} 1$ (most differences likewise being very small). The acquisition data, then, provide no basis for assuming that discrimination between $\mathrm{T} 1$ and $\mathrm{T} 2$ occurred either in Group SL or Group LS. Extinction

Figure 1 shows the extinction speeds for total for each of the three groups on each trial of extinction. The data shown could hardly be more straightforward. Group SL showed increased $R$ to $E$, while Group LS showed no tendency toward greater $R$ to $E$ than Group LL. An analysis of the data shown in Fig. 1 indicated that the differences were highly significant for total $(F=16.80, \mathrm{df}=2 / 33, \mathrm{p}<.001)$, as were the differences in all alley sections.

\section{DISCUSSION}

Group SL showed greater $R$ to $E$ than did Group LS when neither group anticipated smaller reward. This result is not consistent with Lawrence \& Festinger's (1962) view that $R$ to $E$ is regulated by sequence of reward only when smaller reward is anticipated. A second major finding was that Group LS failed to show any increase in $R$ to $E$ relative to Group LL. This result is consistent neither with the overall dissonance hypothesis nor with the frustration hypothesis (e.g., Amsel, 1958). Clearly, Group LS must have experienced some dissonance or frustration in acquisition. In other respects, the acquisition training received by Group LS was also adequate for obtaining increased $R$ to $E$ according to both dissonance and frustration, i.e., following the dissonance-producing or frustrating trials, additional acquisition trials were experienced so that dissonance could be reduced or frustration could be conditioned to the instrumental reaction. The failure of Group LS to show any increase in $R$ to $E$ could mean that frustration or dissonance is irrelevant in terms of increasing $\mathrm{R}$ to $\mathrm{E}$. An alternative view is that, in addition to frustration or dissonance, some other factor(s) must be present in order to increase $\mathrm{R}$ to $\mathrm{E}$ and that this factor(s) has neither been recognized nor identified by either hypothesis. Clear1y, experiencing frustration or dissonance is not enough for increasing $R$ to $\mathrm{E}$.

The specific question under investigation here was, is $R$ to $E$ related to sequence of reward when $S$ receives two different reward magnitudes and no evidence exists that the smaller magnitude is anticipated? The present results suggest an affirmative answer to this question. Lawrence \& Festinger (1962), approaching this issue from the other side, assumed that once smaller reward began to be anticipated, $R$ to $E$ would be reduced, so much so that differences between partial and consistent reinforcement would disappear. This view is not a necessary consequence of the proposition that anticipated smaller reward is not dissonance producing. Another possibility is, for example, that when later in acquisition training the animal begins to anticipate smaller reward, the now-anticipated smaller reward becomes a "null" trial, neither increasing nor decreasing $R$ to $E$. Still another alternative is that the smaller reward that is anticipated increases $R$ to $E$, although perhaps in a different way from when larger reward is anticipated. This alternative, too, is not inconsistent with the view that anticipated smaller reward is not dissonance producing or frustrating, although both Lawrence \& Festinger (1962) and Amsel (1958) have rejected it, i.e., they have assumed that $R$ to $E$ is increased only under expectationdisconfirmation conditions. Moreover, this alternative may be consistent with a variety of recent findings that suggest that it is not necessary that reward be expected on nonrewarded trials in order to increase $R$ to E (McCain, 1966, Robbins, Chait, \& Weinstock, 1968; Spear, Hill, \& O'Sullivan, 1965; Spear \& Spitzner, 1967). In these investigations, increased $R$ to $E$ was obtained even though nonrewarded trials were not preceded by rewarded ones, and in some of these investigations, at least, measures were adopted to minimize or eliminate preexperimental expectancy. In cases of this type, designated as nonanticipated nonreward, reward is not only not expected on the nonrewarded trials, but it never was. ${ }^{2}$ This contrasts with anticipated nonreward, considered earlier, in which reward is not now expected on the nonrewarded trials, but once was (e.g., Capaldi, 1958; MacKinnon, 1967). Anticipated nonreward may well have different effects on $R$ to $E$ than does nonanticipated nonreward. And still different effects may be produced, as Lawrence \& Festinger (1962) and Amsel (1958) suggest, when reward is anticipated but nonreward occurs, a condition that may be called reward-anticipated nonreward. On the other hand, current evidence is compatible with the view that none of these affects $R$ to $E$ differently. That these several kinds of nonreward events differ seems intuitively reasonable. That they differ in such a manner as to affect $R$ to $E$ differentially is a separate issue, although this has not been widely recognized. In any event, a determination of these differences and their effects, if any, on $R$ to $E$ awaits more serious experimental study, a better understanding of the alternatives that face us, clearer specification of the implications of data that are currently available, and, especially, more detailed specification of the sorts of experimental manipulations that can potentially contribute to an understanding of the problem. Some considerations relevant to these matters are advanced in Capaldi, Ziff, \& Godbout (1970).

\section{REFERENCES}

AMSEL, A. The role of frustrative nonreward in noncontinuous reward situations. Psychological Bulletin, 1958, 55, 102-119.

CAPALDI, E. J. The effect of different amounts of training on the resistance to extinction of different patterns of partially reinforced responses. Journal of Comparative \& Physiological Psychology, 1958, 51, 367-371. CAPALDI, E. J. An analysis of the role of reward and reward magnitude in instrumental learning. In J. Reynierse (Ed.), Current issues in animal learning. Lincoln, Nebr.: University of Nebraska Press, in press.

CAPALDI, E. J., ZIFF, D. R., \& GODBOUT, T. Extinction and the necessity or nonnecessity of anticipating reward on nonrewarded trials. Psychonomic Science, 1970, 18, 61-63.

LAWRENCE, D. H., \& FESIINGEK, l. Deterents and reinforcement. Stanford, Calif.: Stanford University Press, 1962.

LEONARD, D. W. Amount and sequence of reward in partial and continuous reinforcement. Journal of Comparative \& Physiological Psychology, 1969, 67, 204-211.

MacKINNON, J. R. Interactive effects of the two rewards in a differential magnitude of reward discrimination. Journal of Experimental Psychology, 1967, 75, 329-338.

McCAIN, G. Partial reinforcement effects following a small number of acquisition trials. Psychonomic Monograph Supplements, 1966, 1 (Whole No. 12), 251-270.

ROBBINS, D., CHAIT, H., \& WEINSTOCK, S. Effects of nonreinforcement on running behavior during acquisition, extinction, and re-acquisition. Journal of Comparative \& Physiological Psychology, 1968, 66, 699-706. 
SPEAR, N. E., Hil L, W. I „ \& O'SULLIVAN, D. J. Acquisition and cxtinction after initial trials without reward. Journal of Experimental Psychology, 1965, 69, 25-29.

SPEAR, N. E. \& SPITZNER, J. H. Effect of initial nonrewarded trials: Factors responsible for increased resistance to extinction. Joumal of Experimental Psychology, 1967, 74, 525-537.

\section{NOTIS}

1. This research was supported in part by National Institute of Child Health amd Human Development Grant HD04379 to the first author.

2. This case could also be called nonanticipated reward, i.e., presumably neither reward nor nonreward is anticipated.

\section{Stimulus novelty as a factor in the intraspecific pain-associated aggression of domesticated rats ${ }^{1}$}

\author{
BENNETT G. GALEF, JR., McMaster \\ University, Hamilton, Ont., Canada
}

Familiar and unfamiliar pairs of male Sprague-Dawley rats were exposed to ascending and descending series of shocks of constant intensity and varying duration. It was found that, while unfamiliar pairs exhibited significantly more aggression than did familiar pairs when subjected to short-duration shocks $(.05$ and .1 sec), there was no difference between the groups at longer shock durations $1.2, .3$, and $.5 \mathrm{sec}$.

Ulrich \& Azrin (1962) have reported that previous familiarity of domestic rats does not affect the frequency of occurrence of intraspecific aggression in response to unavoidable shock. Conversely, the naturally occurring fighting behavior of both domestic (Seward, 1945) and feral (Barnett, 1963; Galef, in press) rats has been found to be affected by the previous familiarity of Ss. In their study, Ulrich and Azrin used a single duration and intensity of shock $(.5 \mathrm{sec}, 1.3 \mathrm{~mA})^{2}$ in testing the effects of $S$ familiarity on intraspecific pain-associated aggression. Wetzel, Conner, \& Levine (1967), working with 85-day postoperative septally lesioned rats and their sham-operated controls, have found a significant Group by Intensity interaction in aggression in response to shock. Whereas there was a large difference between lesion and control groups in probability of fighting at low intensities of shock, the difference was much reduced at high intensities. The present experiment was designed to test the possibility that previous familiarity of Ss might similarly affect the incidence of intraspecific aggression associated with mild shock but not with intense shock.

Because Dreyer \& Church (1968) have shown that the slope of the line describing incidence of aggression as a function of increasing intensity of shock is far steeper than that describing incidence of aggression as a function of shock duration, it was decided to use duration as an independent variable in the present experiment and to hold intensity constant. A broad range of shocks eliciting moderate frequencies of aggression were thus available for presentation.

\section{SUBJECTS}

The Ss were 40 male Sprague-Dawley rats, averaging $253 \mathrm{~g}$ at the outset of the experiment. Data from one $S$ was discarded because he showed seizures in response to painful stimulation.

\section{PROCEDURE}

The Ss were confined in pairs for either 3 or 6 weeks in small hanging cages ( $7 \times 9 \frac{1 / 2}{2} \times 7$ in.). The procedure described below was carried out twice, once for the 3-week confinement group (20 Ss) and once for the 6-week confinement group $(20 \mathrm{Ss})$. Following its period of confinement, each $S$ was placed in a 6-in.-square shockbox and exposed to two shock sessions, one with its familiar cage-mate and one with an unfamiliar $S$ from another hanging cage. Forty-eight hours separated the two shock sessions for any given $S$. Half of the Ss were shocked first in the presence of an unfamiliar partner, and half were shocked first in the presence of their familiar cage-mate.

Prior to shock initiation, each pair of Ss was allowed $3 \mathrm{~min}$ to become accustomed to the shockbox. Alternating ascending and descending series of shocks, .05, .1, .2, .3 , and $.5 \mathrm{sec}$ in duration and $1.3 \mathrm{~mA}$ in intensity, were then delivered, 12 shocks per minute, through the floor of the

Table 1

Percentage of Shock Trials on Which Fighting was Observed

\begin{tabular}{|c|c|c|c|c|c|}
\hline \multirow[b]{2}{*}{ Group } & \multicolumn{5}{|c|}{ Shock Duration (Sec) } \\
\hline & .05 & .1 & .2 & .3 & .5 \\
\hline $\begin{array}{l}3 \text { Wk. Familiar } \\
3 \text { Wk. Unfamiliar }\end{array}$ & $\begin{array}{l}.27 \\
.35^{*}\end{array}$ & $\begin{array}{l}.38 \\
.50^{*}\end{array}$ & $\begin{array}{l}.56 \\
.56\end{array}$ & $\begin{array}{l}.64 \\
.63\end{array}$ & $\begin{array}{l}.70 \\
.69\end{array}$ \\
\hline $\begin{array}{l}6 \text { Wk. Familiar } \\
6 \text { Wk. Unfamiliar }\end{array}$ & $\begin{array}{l}.23 \\
.43^{* *}\end{array}$ & $\begin{array}{l}.38 \\
.54^{*}\end{array}$ & $\begin{array}{l}.53 \\
.60\end{array}$ & $\begin{array}{l}.63 \\
.70\end{array}$ & $\begin{array}{l}.70 \\
.78\end{array}$ \\
\hline
\end{tabular}

$* p<.05 \quad * * 0<.01$ shockbox via a Grason-Stadler Model E1064GS shock generator and scrambler. Each pair of Ss received 20 alternating ascending and descending serjes of shocks, a total of 40 shocks of each duration.

The $\mathrm{E}$ recorded the presence or absence of fighting following each shock presentation. A fight was recorded whenever both Ss were in an upright posture and one lunged at or made contact with the other (Dreyer \& Church, 1968). The experiment was run blind so that $E$ did not know whether any given pair in the shockbox was from the same or from different hanging cages.

\section{RESULTS AND DISCUSSION}

The results are presented in Table 1. Whereas Kolmogorov-Smirnov two-sample tests showed significant differences between familiar and unfamiliar pairs in the percentage of trials on which fighting occurred at short shock duration (.05 and $.1 \mathrm{sec})$ for both the 3- and 6-week familiarization groups, there was no significant difference in percentage of fights between familiar and unfamiliar pairs using longer shock durations $(.2, .3$, and $.5 \mathrm{sec}$ ).

The data indicate that, within a limited range of aversive stimulation, opponent novelty is an important variable in determining the probability of occurrence of shock-associated intraspecific aggression. The results are in accord with those of Ulrich \& Azrin (1962) in that, using their shock parameters, no significant effect of opponent familiarity was observed.

The results-further suggest that the general importance of opponent or target variables in eliciting aggression in pain-induced aggression situations may be a function of the degree of noxious stimulation applied.

\section{REFERENCES}

BARNETT, S. A. The rat, a study in behavior. Chicago: Aldine, 1963.

DREYER, P. I., \& CHURCH, R. Shock-induced fighting as a function of the intensity and duration of the aversive stimulus. Psychonomic Science, 1968, 10, 271-272.

GALEF, JR., B. G. Aggression and timidity: Responses to novelty in feral Norway rats. Journal of Comparative \& Physiological Psychology, in press.

SEWARD, J. P. Aggressive behavior in the rat. Journal of Comparative $\&$ Physiological Psychology, 1945, 38, 175-197.

ULRICH, R. E., \& AZRIN, N. H. Reflexive fighting in response to aversive stimulation. Joumal of the Experimental Analysis of Behavior, 1962, 5, 511-520.

WETZEL, A. B., CONNER, R. L., \& LEVINE, S. Shock induced fighting in septal lesioned rats. Psychonomic Science, 1967, 9, 133-134. NOTES

1. This research was supported by Grants AP307 and APA0307 of the National Research Council of Canada. Susan McLellan performed the experimental testing.

2. Ulrich, R. E., personal communication. 